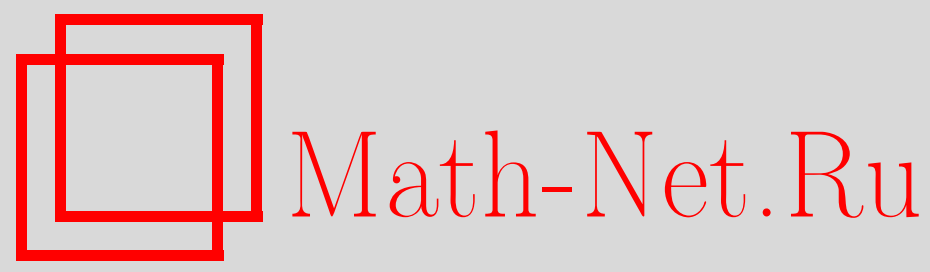

Ю. Н. Субботин, С. А. Теляковский, Точные значения относительных поперечников классов дифференцируемых функций, Матем. заметки, 1999, том 65, выпуск 6, 871879

DOI: https://doi.org/10.4213/mzm1123

Использование Общероссийского математического портала Math-Net.Ru подразумевает, что вы прочитали и согласны с пользовательским соглашением http://www.mathnet.ru/rus/agreement

Параметры загрузки:

IP : 54.209 .52 .79

26 апреля 2023 г., $14: 29: 58$

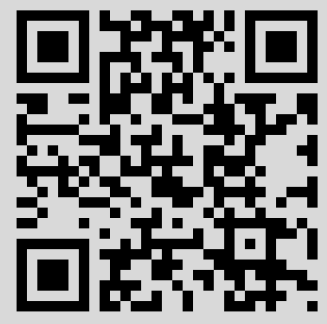




\title{
ТОЧНЫЕ ЗНАЧЕНИЯ ОТНОСИТЕЛЬНЫХ ПОПЕРЕЧНИКОВ КЛАССОВ ДИФФЕРЕНЦИРУЕМЫХ ФУНКЦИЙ
}

\author{
Ю.Н. Субботин, С. А. Теляковский
}

Изучаются относительные поперечники в пространствах $C$ и $L$ классов периодических дифференцируемых функций $W^{r}, r=1,2, \ldots$, когда в отличие от колмогоровских поперечников дополнительно требуется, чтобы приближающие функции принадлежали классу $M W^{r}$ с заданной мажорантой $M$ нормы производной порядка $r$. Доказано, что если для $M$ вьполнена равномерная по $n$ и $r$ оценка

$$
M \geqslant \frac{4}{\pi^{2}} \log \min (n, r)+O(1)
$$

то указанные $n$-мерные относительные поперечники классов $W^{r}$ совпадают с колмогоровскими поперечниками.

Попутно получена равномерная по всем параметрам оценка констант Лебега нормальных средних Зигмунда рядов Фурье, определяемых множителями $1-(k / n)^{r}$, $k \leqslant n$.

Библиография: 10 названий.

1. Введение. Понятие относительного поперечника введено В. Н. Коноваловым [1]. Пусть $X$ - банаховопространство, $W$ и $V$ - центрально симметричные подмножества $X$. Относительным п-мерным поперечником мнохсества $W$ относительно мнохсества $V$ в пространстве $X$ назьвается величина

$$
K_{n}(W, V, X):=\inf _{L_{n}} \sup _{f \in W} \inf _{g \in L_{n} \cap V}\|f-g\|_{X},
$$

где $L_{n}$ - линейные подпространства $X$ размерности $n$.

Здесь в отличие от классических колмогоровских поперечников требуется, чтобы приближающие элементы $g$ из $L_{n}$ принадлежали множеству $V$. В этой терминологии колмогоровские поперечники соответствуют случаю $V=X$. Как обычно, будем обозначать их через $d_{n}(W, X)$.

Обозначим через $M W_{C}^{r}, r=1,2, \ldots$, класс $2 \pi$-периодических функций $f$, удовлетворяющих условию $\left|f^{(r-1)}\left(x_{1}\right)-f^{(r-1)}\left(x_{2}\right)\right| \leqslant M\left|x_{1}-x_{2}\right|$. Если $M=1$, то будем писать $W_{C}^{r}$.

Коновалов [1] доказал, что для $r=1$ и $r=2$ поперечники $K_{n}\left(W_{C}^{r}, W_{C}^{r}, C\right)$ имеют тот же порядок убывания при $n \rightarrow \infty$, что и $d_{n}\left(W_{C}^{r}, C\right)$, а для $r \geqslant 3$ точньй порядок

Работа выполнена при финансовой поддержке совместной программы Российского фонда фундаментальных исследований и ГФЕН Китая, грант № 96-01-00036С. 
величин $K_{n}\left(W_{C}^{r}, W_{C}^{r}, C\right)$ равен $n^{-2}$. В [2] В.М. Тихомиров дал другое доказательство этих результатов (где рассматривались классы $W_{C}^{r}$ и для нецелых $r$ ).

Отметим, что при $r=1$ и $r=2$ для четных $n$ справедливо равенство

$$
K_{n}\left(W_{C}^{r}, W_{C}^{r}, C\right)=d_{n}\left(W_{C}^{r}, C\right)
$$

Для $r=1$ равенство (1) вытекает из результатов В. Н. Малоземова [3, теорема 1] о приближении функций ломаными, а для $r=2$ оно доказано Ю. Н. Субботиным [4, теорема 2].

Мы рассматриваем случай, когда $r \geqslant 3$.

В [5] В. $\Phi$. Бабенко при $r=3,4, \ldots$ оценил сверху относительные поперечники

$$
K_{n}\left(W_{C}^{r},\left(1+\varepsilon_{n}\right) W_{C}^{r}, C\right)
$$

где $\left\{\varepsilon_{n}\right\}$ - заданная последовательность положительных чисел. Из его результатов, в частности, следует, что для фиксированного положительного числа $\varepsilon$ имеет место порядковое равенство

$$
K_{n}\left(W_{C}^{r},(1+\varepsilon) W_{C}^{r}, C\right) \sim n^{-r}, \quad r=3,4, \ldots
$$

которое в качестве гипотезы было высказано С. Б. Стечкиньм (см. [5, с. 25]).

Ясно, что если взять $M=M(n, r)$ достаточно большим, то справедливо равенство

$$
K_{n}\left(W_{C}^{r}, M W_{C}^{r}, C\right)=d_{n}\left(W_{C}^{r}, C\right)
$$

В настоящей работе дается оценка значений $M$, при которых это равенство имеет место. Параллельно мы рассматриваем аналогичную задачу об относительньх поперечниках соответствующих классов функций в пространстве $L$.

Обозначим через $M W_{L}^{r}, r=1,2, \ldots$, класс $2 \pi$-периодических функций $f$, у которых вариация производной $f^{(r-1)}$ на периоде ограничена числом $M$. При $M=1$ будем писать $W_{L}^{r}$.

Относительным поперечникам классов $W_{L}^{r}$ посвящены работы Бабенко [6], [7]. В [6] для $K_{n}\left(W_{L}^{r}, W_{L}^{r}, L\right)$ установлены оценки, аналогичные результатам Коновалова для $K_{n}\left(W_{C}^{r}, W_{C}^{r}, C\right)$, и доказано, что для четных $n$ при $r=1$ и $r=2$ справедливо равенство

$$
K_{n}\left(W_{L}^{r}, M W_{L}^{r}, L\right)=d_{n}\left(W_{L}^{r}, L\right)
$$

А в [7] для поперечников $K_{n}\left(W_{L}^{r},\left(1+\varepsilon_{n}\right) W_{L}^{r}, L\right)$ получены такие же результаты, как в [5] для $K_{n}\left(W_{C}^{r},\left(1+\varepsilon_{n}\right) W_{C}^{r}, C\right)$.

\section{2. Основные результаты.}

Tеорема 1. Существует абсолютная постоянная $A$ такая, что для

$$
M \geqslant \frac{4}{\pi^{2}} \log \min (m, r)+A
$$

справедливы равенства (2) и (3). 
ДоКАЗАТЕЛЬСТво. ИЗвестно [8, теоремы 10.4.1, 10.5.1 и 5.5.1], что колмогоровские поперечники $d_{n}\left(W_{C}^{r}, C\right)$ и $d_{n}\left(W_{L}^{r}, L\right)$ для $n=2 m-1$ и $n=2 m, m=1,2, \ldots$, достигаются при приближении функций $f$ средними Фавара - полиномами

$$
u_{m}(f, x, \lambda)=\frac{a_{0}}{2}+\frac{1}{\pi} \int_{0}^{2 \pi} \sum_{k=1}^{m-1} \frac{\lambda_{k}(r)}{k^{r}} \cos \left(k(x-t)-\frac{r \pi}{2}\right) d f^{(r-1)}(t),
$$

где $\lambda_{k}(r)$ определяются для нечетных $r$ равенствами

$$
\lambda_{k}(r):=1-\sum_{s=1}^{\infty}\left(\left(\frac{k}{2 s m-k}\right)^{r}-\left(\frac{k}{2 s m+k}\right)^{r}\right)
$$

и для четных $r$ равенствами

$$
\lambda_{k}(r):=1-\sum_{s=1}^{\infty}(-1)^{s+1}\left(\left(\frac{k}{2 s m-k}\right)^{r}+\left(\frac{k}{2 s m+k}\right)^{r}\right) .
$$

При этом

$$
d_{2 m-1}\left(W_{C}^{r}, C\right)=d_{2 m}\left(W_{C}^{r}, C\right)=d_{2 m-1}\left(W_{L}^{r}, L\right)=d_{2 m}\left(W_{L}^{r}, L\right)=\frac{\mathscr{K}_{r}}{m^{r}},
$$

где $\mathscr{K}_{r}-$ константы $\Phi$ авара

$$
\mathscr{K}_{r}=\frac{4}{\pi} \sum_{k=0}^{\infty} \frac{(-1)^{k(r+1)}}{(2 k+1)^{r+1}} .
$$

Для поперечников в $C$ это доказал В.М. Тихомиров, значения $d_{n}\left(W_{L}^{r}, L\right)$ для нечетных $n$ нашли независимо Ю.И. Маковоз и Ю.Н. Субботин, а для четных $n-$ В.И. Рубан.

Для производных порядка $r$ полиномов $u_{m}(f, x, \lambda)$ имеет место представление

$$
u_{m}^{(r)}(f, x, \lambda)=\frac{1}{\pi} \int_{0}^{2 \pi} \sum_{k=1}^{m-1} \lambda_{k}(r) \cos k(x-t) d f^{(r-1)}(t) .
$$

Поэтому, полагая

$$
I(m, r):=\frac{2}{\pi} \int_{0}^{\pi}\left|\sum_{k=1}^{m-1} \lambda_{k}(r) \cos k x\right| d x,
$$

получим неравенство для $f \in W_{C}^{r}$

$$
\left\|u_{m}^{(r)}(f, x, \lambda)\right\|_{C} \leqslant I(m, r)
$$

и аналогичное неравенство для $f \in W_{L}^{r}$

$$
\left\|u_{m}^{(r)}(f, x, \lambda)\right\|_{L} \leqslant I(m, r) .
$$

Это показывает, что если $M \geqslant I(m, r)$, то справедливы равенства (2) и (3). Таким образом, теорема будет доказана, если мы установим следующее предложение. 
ЛЕмма. Для любых натуральных $m$ и $г$ справедлива равномерная по этим параметрам оченка

$$
I(m, r)=\frac{4}{\pi^{2}} \log \min (m, r)+O(1) .
$$

Отметим, что здесь и далее все оценки с $O$-символами являются равномерными относительно всех параметров.

Доказательству леммы посвящены п. 3-5 настоящей статьи. При этом мы будем оценивать не интеграл (6), а следующий:

$$
I^{*}(m, r):=\frac{2}{\pi} \int_{0}^{\pi}\left|\frac{\lambda_{0}(r)}{2}+\sum_{k=1}^{m-1} \lambda_{k}(r) \cos k x\right| d x
$$

где числа $\lambda_{0}(r)$ так же, как и $\lambda_{k}(r)$ при $k \geqslant 1$, определяются соответственно формулами (4) и (5). Заметим, что $\lambda_{0}(r)=1$ для всех $r$. Поэтому достаточно доказать оценку вида (7) для интеграла (8).

3. Оценки норм тригонометрических полиномов. Мы будем пользоваться следующей оценкой, установленной Стечкиньм [9, теорема 2]. Если числа $\lambda_{0}, \lambda_{1}, \ldots$, $\lambda_{m-1}, \lambda_{m}=0$ образуют убьвающую вогнутую последовательность (последнее условие означает, что $\lambda_{k}-2 \lambda_{k+1}+\lambda_{k+2} \leqslant 0$ для $\left.k=0,1, \ldots, m-2\right)$, то

$$
\int_{0}^{\pi}\left|\frac{\lambda_{0}}{2}+\sum_{k=1}^{m-1} \lambda_{k} \cos k x\right| d x=\frac{2}{\pi} \sum_{k=1}^{m-1} \frac{\lambda_{k}}{m-k}+O\left(\lambda_{0}\right) .
$$

Из сказанного ниже станет ясно, что интегралы (8) мало отличаются от констант Лебега нормальных средних Зигмунда

$$
Z(m, r):=\frac{2}{\pi} \int_{0}^{\pi}\left|\frac{z_{0}(r)}{2}+\sum_{k=1}^{m-1} z_{k}(r) \cos k x\right| d x
$$

где

$$
z_{k}(r):=1-\left(\frac{k}{m}\right)^{r}, \quad k=0,1, \ldots, m .
$$

Поэтому в качестве первого шага в доказательстве леммы получим оценку величин $Z(m, r)$.

ТЕорема 2. Для любых натуральных $m$ и $r$ справедлива равномерная по этим параметрам оченка

$$
Z(m, r)=\frac{4}{\pi^{2}} \log \min (m, r)+O(1)
$$

ДокАЗАТЕЛЬСТво. Убывание и вогнутость последовательности $z_{0}(r), z_{1}(r), \ldots$, $z_{m}(r)=0$ очевидны. Поэтому для оценки интеграла (10) можно пользоваться оценкой $(9)$, согласно которой

$$
Z(m, r)=\frac{4}{\pi^{2}} A(m, r)+O(1)
$$

где

$$
A(m, r):=\sum_{k=1}^{m-1} \frac{1}{m-k}\left(1-\left(\frac{k}{m}\right)^{r}\right) .
$$


Представим величину $A(m, r)$ следующим образом:

$$
A(m, r)=\sum_{k=1}^{m-1} \frac{1}{m-k}\left(1-\frac{k}{m}\right) \sum_{i=0}^{r-1}\left(\frac{k}{m}\right)^{i}=\sum_{i=0}^{r-1} \frac{1}{m^{i+1}} \sum_{k=1}^{m-1} k^{i} .
$$

Для сумм

$$
B(m, i):=\sum_{k=1}^{m-1} k^{i}, \quad i=0,1, \ldots
$$

будем пользоваться оценками

$$
\frac{1}{i+1} m^{i+1}-m^{i} \leqslant B(m, i)<\frac{1}{i+1} m^{i+1},
$$

а если $i>m$, то

$$
B(m, i)<2(m-1)^{i} .
$$

Неравенства (14) легко доказываются с помощью перехода к интегралам:

$$
\begin{aligned}
& B(m, i)<\int_{0}^{m} u^{i} d u=\frac{1}{i+1} m^{i+1} \\
& B(m, i)=\sum_{k=1}^{m} k^{i}-m^{i} \geqslant \int_{0}^{m} u^{i} d u-m^{i}=\frac{1}{i+1} m^{i+1}-m^{i}
\end{aligned}
$$

Далее,

$$
B(m, i)<(m-1)^{i}+\int_{0}^{m-1} u^{i} d u=(m-1)^{i}+\frac{1}{i+1}(m-1)^{i+1},
$$

откуда для $i>m$ следует (15).

Применяя к представлению (13) неравенства (14), находим

$$
A(m, r)=\sum_{i=0}^{r-1} \frac{1}{m^{i+1}}\left(\frac{1}{i+1} m^{i+1}+O\left(m^{i}\right)\right)=\log r+O(1)+O\left(\frac{r}{m}\right) .
$$

Значит, если $r \leqslant m$, то

$$
A(m, r)=\log r+O(1) .
$$

Для $r>m$ согласно (13) имеем

$$
A(m, r)=\sum_{i=0}^{m-1} \frac{1}{m^{i+1}} B(m, i)+\sum_{i=m}^{r-1} \frac{1}{m^{i+1}} B(m, i) .
$$

Первая сумма из правой части оценки (17) в силу (16) есть $\log m+O(1)$. А для второй суммы из правой части (17) справедлива оценка, при доказательстве которой используется (15):

$$
\begin{aligned}
\sum_{i=m}^{r-1} \frac{1}{m^{i+1}} B(m, i) & <\sum_{i=m}^{r-1} \frac{1}{m^{i+1}} 2(m-1)^{i}<\frac{2}{m}\left(\frac{m-1}{m}\right)^{m} \sum_{i=0}^{\infty}\left(\frac{m-1}{m}\right)^{i} \\
& <\frac{1}{m} \cdot \frac{1}{1-(m-1) / m}=1
\end{aligned}
$$


Поэтому при $r>m A(m, r)=\log m+O(1)$. Отсюда и из (16) вытекает оценка

$$
A(m, r)=\log \min (m, r)+O(1)
$$

которая в силу (12) приводит к (11). Теорема доказана.

Ранее была известна ограниченность чисел $Z(m, r)$ величиной, зависящей только от $r$ (см. [10, с. 46]). Теорема 2 уточняет этот результат.

4. Оценка интеграла (8) для коэффициентов (4). Покажем сначала, что при каждом $m$ последовательность $\lambda_{0}(r), \lambda_{1}(r), \ldots, \lambda_{m}(r)$, задаваемая формулой (4), убывает и вогнута. При этом будем пользоваться следующим представлением чисел (4):

$$
\lambda_{k}(r)=1+\sum_{s=1}^{\infty}\left(\left(\frac{k}{2 s m+k}\right)^{r}-\left(\frac{k}{2 s m-k}\right)^{r}\right)=1+\sum_{s=1}^{\infty} \varphi_{s}(k)
$$

где

$$
\varphi_{s}(x):=\left(\frac{x}{2 s m+x}\right)^{r}-\left(\frac{x}{2 s m-x}\right)^{r} .
$$

Так как для $x \in(0, m]$

$$
\varphi_{s}^{\prime}(x)=2 s m r x^{r-1}\left(\frac{1}{(2 s m+x)^{r+1}}-\frac{1}{(2 s m-x)^{r+1}}\right)<0,
$$

числа $\left\{\lambda_{k}(r)\right\}$ с ростом $k$ убывают. Далее, для таких $x$

$$
\begin{aligned}
\varphi_{s}^{\prime \prime}(x)= & 2 s m r(r-1) x^{r-2}\left(\frac{1}{(2 s m+x)^{r+1}}-\frac{1}{(2 s m-x)^{r+1}}\right) \\
& -2 s m r(r+1) x^{r-1}\left(\frac{1}{(2 s m+x)^{r+2}}+\frac{1}{(2 s m-x)^{r+2}}\right)<0 .
\end{aligned}
$$

Значит, последовательность $\lambda_{0}(r), \lambda_{1}(r), \ldots, \lambda_{m}(r)$ вогнута.

Таким образом, к интегралу $I^{*}(m, r)$ применима оценка $(9)$.

При оценке суммы

$$
\sum_{k=1}^{m-1} \frac{\lambda_{k}(r)}{m-k}
$$

воспользуемся тем, что

$$
\sum_{s=1}^{\infty}\left(\frac{1}{(2 s m-m)^{r}}-\frac{1}{(2 s m+m)^{r}}\right)=\frac{1}{m^{r}}
$$

Поэтому

$$
\begin{aligned}
\lambda_{k}(r)= & -\left(\frac{k}{m}\right)^{r}+k^{r} \sum_{s=1}^{\infty}\left(\left(\frac{1}{(2 s m-m)^{r}}-\frac{1}{(2 s m+m)^{r}}\right)\right. \\
& \left.-\left(\frac{1}{(2 s m-k)^{r}}-\frac{1}{(2 s m+k)^{r}}\right)\right) .
\end{aligned}
$$


Вклад в сумму (19) от выражения $1-(k / m)^{r}$ оценен при доказательстве теоремы 2 : это величина $A(m, r)$.

Рассмотрим последнее выражение в представлении $(20)$ чисел $\lambda_{k}(r)$. Имеем

$$
\begin{aligned}
\alpha_{k} & :=\left|\sum_{s=1}^{\infty}\left(\left(\frac{k^{r}}{(2 s m-m)^{r}}-\frac{k^{r}}{(2 s m+m)^{r}}\right)-\left(\frac{k^{r}}{(2 s m-k)^{r}}-\frac{k^{r}}{(2 s m+k)^{r}}\right)\right)\right| \\
& \leqslant k^{r} \sum_{s=1}^{\infty}\left(\left|\frac{1}{(2 s m-m)^{r}}-\frac{1}{(2 s m-k)^{r}}\right|+\left|\frac{1}{(2 s m+m)^{r}}-\frac{1}{(2 s m+k)^{r}}\right|\right) .
\end{aligned}
$$

Применяя формулу конечных приращений Лагранжа, видим, что

$$
\begin{aligned}
& \left|\frac{1}{(2 s m-m)^{r}}-\frac{1}{(2 s m-k)^{r}}\right|<\frac{r(m-k)}{(2 s m-m)^{r+1}} \\
& \left|\frac{1}{(2 s m+m)^{r}}-\frac{1}{(2 s m+k)^{r}}\right|<\frac{r(m-k)}{(2 s m)^{r+1}} .
\end{aligned}
$$

Поэтому

$$
\alpha_{k}<k^{r} r(m-k) \sum_{p=1}^{\infty} \frac{1}{(p m)^{r+1}}<\frac{2 r(m-k)}{m^{r+1}} k^{r} .
$$

Отсюда с помощью правого неравенства (14) находим

$$
\sum_{k=1}^{m-1} \frac{\alpha_{k}}{m-k}<2 r \sum_{k=1}^{m-1} \frac{k^{r}}{m^{r+1}}<2
$$

Из (20), (18) и (22) вытекает оценка суммы (19):

$$
\sum_{k=1}^{m-1} \frac{\lambda_{k}(r)}{m-k}=\log \min (m, r)+O(1)
$$

Таким образом, согласно (9) для коэффициентов $\lambda$, задаваемьх формулой (4), лемма доказана.

5. Оценка интеграла (8) для коэффициентов (5). Рассуждения проведем по той же схеме, что и для коэффициентов, задаваемых формулой (4). Покажем, что последовательность $\lambda_{0}(r), \lambda_{1}(r), \ldots, \lambda_{m}(r)$ коэффициентов (5) убывает и вогнута.

Запишем числа $\lambda_{k}(r)$ следуюшим образом:

$$
\lambda_{k}(r)=1-\sum_{p=1}^{\infty} \psi_{p}(k)
$$

где

$$
\psi_{p}(x):=\left(\frac{x}{(4 p-2) m-x}\right)^{r}+\left(\frac{x}{(4 p-2) m+x}\right)^{r}-\left(\frac{x}{4 p m-x}\right)^{r}-\left(\frac{x}{4 p m+x}\right)^{r} .
$$


Будем рассматривать $x \in(0, m]$. Имеем $\psi_{p}^{\prime}(x)=r x^{r-1} \sigma_{1}+r x^{r} \sigma_{2}$, где

$$
\begin{aligned}
\sigma_{1} & :=\frac{1}{((4 p-2) m-x)^{r}}+\frac{1}{((4 p-2) m+x)^{r}}-\frac{1}{(4 p m-x)^{r}}-\frac{1}{(4 p m+x)^{r}}, \\
\sigma_{2} & :=\frac{1}{((4 p-2) m-x)^{r+1}}-\frac{1}{((4 p-2) m+x)^{r+1}}-\frac{1}{(4 p m-x)^{r+1}}+\frac{1}{(4 p m+x)^{r+1}} .
\end{aligned}
$$

Оценка $\sigma_{1}>0$ очевидна. Неравенство $\sigma_{2}>0$ вытекает из того, что разность

$$
\frac{1}{(a-x)^{r+1}}-\frac{1}{(a+x)^{r+1}}, \quad a>x,
$$

как функция от $a$ убывает, в чем легко убедиться, рассмотрев ее производную по $a$.

Таким образом, $\psi_{p}^{\prime}(x)>0$, откуда в силу (24) следует, что числа $\lambda_{k}(r)$ с ростом $k$ убывают.

Оценим теперь знак второй производной функций $\psi_{p}(x)$. При этом будем пользоваться представлением

$$
\psi_{p}^{\prime \prime}(x)=r(r-1) x^{r-2} \sigma_{1}+2 r^{2} x^{r-1} \sigma_{2}+r(r+1) \sigma_{3}
$$

где $\sigma_{1}$ и $\sigma_{2}$ те же, что и вьше, а

$$
\sigma_{3}:=\frac{1}{((4 p-2) m-x)^{r+2}}+\frac{1}{((4 p-2) m+x)^{r+2}}-\frac{1}{(4 p m-x)^{r+2}}-\frac{1}{(4 p m+x)^{r+2}}
$$

Положительность величин $\sigma_{1}$ и $\sigma_{2}$ нам уже известна, а оценка $\sigma_{3}>0$ очевидна. Поэтому из (25) следует неравенство $\psi_{p}^{\prime \prime}(x)>0$, которое доказывает вогнутость последовательности $\lambda_{0}(r), \lambda_{1}(r), \ldots, \lambda_{m}(r)$.

Значит, для оценки интеграла $I^{*}(m, r)$ можно пользоваться формулой $(9)$.

Рассмотрим сумму

$$
\sum_{k=1}^{m-1} \frac{\lambda_{k}(r)}{m-k}
$$

Так как

$$
\sum_{s=1}^{\infty}(-1)^{s+1}\left(\frac{1}{(2 s m-m)^{r}}+\frac{1}{(2 s m+m)^{r}}\right)=\frac{1}{m^{r}}
$$

то

$$
\begin{aligned}
\lambda_{k}(r)= & -\left(\frac{k}{m}\right)^{r}+\sum_{s=1}^{\infty}(-1)^{s+1}\left(\left(\frac{k^{r}}{(2 s m-m)^{r}}+\frac{k^{r}}{(2 s m+m)^{r}}\right)\right. \\
& \left.-\left(\frac{k^{r}}{(2 s m-k)^{r}}+\frac{k^{r}}{(2 s m+k)^{r}}\right)\right) .
\end{aligned}
$$

Вклад в сумму (26) от выражения $1-(k / m)^{r}$ есть величина $A(m, r)$. 
Оценивая выражение

$$
\begin{aligned}
\beta_{k} & :=\left|\sum_{s=1}^{\infty}(-1)^{s+1}\left(\left(\frac{k^{r}}{(2 s m-m)^{r}}+\frac{k^{r}}{(2 s m+m)^{r}}\right)-\left(\frac{k^{r}}{(2 s m-k)^{r}}+\frac{k^{r}}{(2 s m+k)^{r}}\right)\right)\right| \\
& \leqslant k^{r} \sum_{s=1}^{\infty}\left(\left|\frac{1}{(2 s m-m)^{r}}-\frac{1}{(2 s m-k)^{r}}\right|+\left|\frac{1}{(2 s m+m)^{r}}-\frac{1}{(2 s m+k)^{r}}\right|\right)
\end{aligned}
$$

видим, что для чисел $\beta_{k}$ справедлива такая же оценка, как оценка (21) для чисел $\alpha_{k}$. Поэтому имеют место оценки

$$
\sum_{k=1}^{m-1} \frac{\beta_{k}}{m-k}<2
$$

и

$$
\sum_{k=1}^{m-1} \frac{\lambda_{k}(r)}{m-k}=\log \min (m, r)+O(1),
$$

аналогичные (22) и (23) соответственно. Таким образом, лемма доказана и для коэффициентов (5).

\section{СПИСОК ЦИТИРОВАННОЙ ЛИТЕРАТУРЫ}

[1] Коновалов В.Н. Оценки поперечников типа Колмогорова для классов дифференцируемых периодических функций // Матем. заметки. 1984. Т. 35. №3. С. 369-380.

[2] Tikhomirov V. M. Some remarks on relative diameters // Banach Center Publ. 1989. V. 22. P. 471-474.

[3] Малоземов В.Н. Об отклонении ломаных // Вестн. ЛГУ. Матем. , мех. , астрон. 1966. № 7. C. $150-153$.

[4] Субботин Ю.Н. Наследование свойств монотонности и выпуклости при локальной аппроксимации // ЖВМиМФ. 1993. Т. 33. С. 996-1003.

[5] Бабенко В.Ф. О наилучших равномерных приближениях сплайнами при наличии ограничений на их производные // Матем. заметки. 1991. Т. 50. №6. С. 24-30.

[6] Бабенко В.Ф. Приближения в среднем при наличии ограничений на производные приближаюших функций // Вопросы анализа и приближения. Сб. научн. тр. Киев: ИМ АН УССР, 1989. C. $9-18$.

[7] Бабенко В. Ф. О наилучших $L_{1}$-приближениях сплайнами при наличии ограничений на их производные // Матем. заметки. 1992. Т. 51. № 5. С. 12-19.

[8] Корнейчук Н. П. Экстремальные задачи теории приближений. М.: Наука, 1976.

[9] Стечкин С. Б. К проблеме множителей для тригонометрических полиномов // Докл. АН CCCP. 1950. T. 75 . C. $165-168$.

[10] Sz.-Nagy B. Sur une classe générale de procédés de sommation pour les séries de Fourier // Hungar. Acta Math. 1948. V. 1. № 3. P. 14-52. 